\title{
Diseño e implementación de un Plan de Manejo para el Sistema de Humedales de la Bahía de Bluefields, Nicaragua: En la búsqueda del manejo sostenible en áreas marino-costeras
}

Milton Saúl Castrillo López ${ }^{1}$

Recibido: Julio 2004 / Aceptado: Octubre 2004

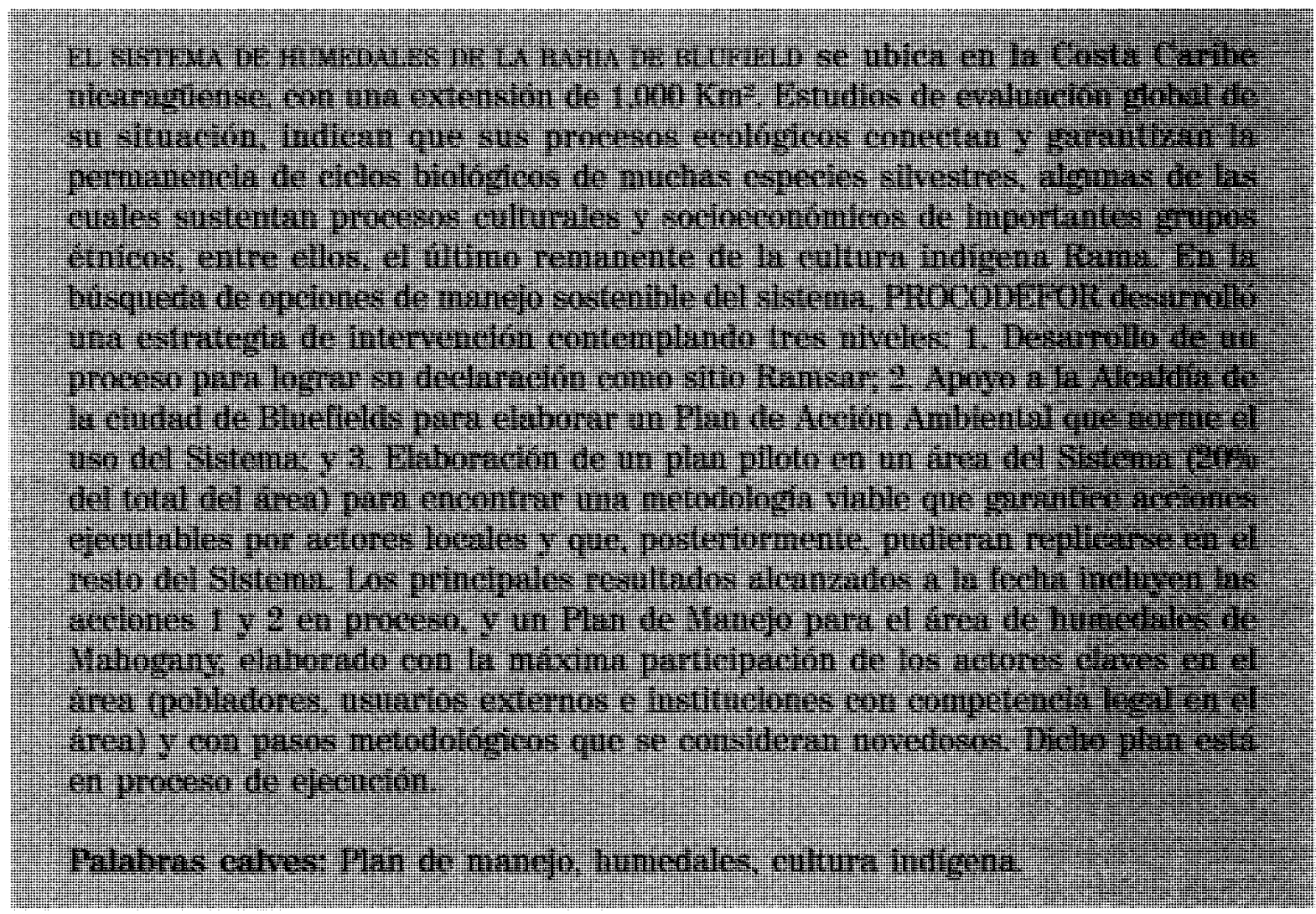




\section{Introducción}

El Sistema de Humedales de la Bahía de Bluefields está conformado por diversos ecosistemas desde salados y salobres a dulceacuícolas, distribuidos en el entorno de la Bahía, que en realidad es una laguna costera. El Sistema se extiende en un área de un poco más de $1000 \mathrm{Km}^{2}$. Las cuencas que drenan sobre el Sistema son las del Río Escondido y la del Río Kukra, con un área total de $12,700 \mathrm{~km}^{2}$. Los aportes de aguas oceánicas llegan por medio de dos bocas: el Bluff y Hone Sound. Las principales formaciones vegetales presentes son: las llanuras de inundación, los bosques pantanosos y los manglares, que proveen áreas para reproducción, crianza y dispersión a fauna acuática y terrestre. En el área se asienta una población multiétnica (Creoles, Ramas y mestizos principalmente). Los principales procesos naturales que se dan en el sistema sustentan pesquerías artesanales (peces, camarones, langostas, ostras, cangrejos y otros), que son la base económica y cultural de los grupos étnicos, especialmente del grupo Rama. La mayor parte del Sistema se encuentra dentro del área reconocida como Reserva Natural Cerro Silva y se está regenerando después de ser devastado por el huracán "Juana” en 1988. En la actualidad, la zona tiene problemas de contaminación y como consecuencia de los efectos del aumento poblacional, principalmente traducidos en incendios continuos y deforestación, que han afectado de manera drástica sus funciones y atributos.

\section{Metodología}

Descripción del proceso de intervención en el área: En el Sistema de Humedales de la Bahía de Bluefields, se reconocen los mismos problemas inherentes a la viabilidad de ejecución de planes de manejo, comunes en los países del Tercer Mundo, como consecuencia de la poca capacidad estatal (presupuestaria, técnica, política) y local (capacidad técnica y económica principalmente). En este caso, el problema se trató de abordar desde tres ángulos:

1. Facilitar el acceso a recursos financieros externos con la declaración del Sistema como sitio Ramsar.

2. Creando, con un actor clave, las condiciones básicas de control del uso del área mediante la elaboración de un Plan de Acción Ambiental regido por la Municipalidad de Bluefields. Este Plan entra dentro del andamiaje de Planificación Estratégica de esta Municipalidad.

3. La búsqueda de una metodología de planificación del uso y manejo del área que, a diferencia de otras experiencias, llevará dentro de sí la posibilidad de permanecer en el tiempo sin mucho apoyo externo.

Los dos primeros pasos intentan crear el marco legal y normativo que facilitará la ejecución de acciones en el territorio y el acceso a recursos financieros. Se consideró que la fase más crítica en el proceso consistió en el diseño de una metodología de elaboración de 
planes de manejo que garantizara la participación real de los diferentes actores locales con sus conocimientos, competencias e intereses; conjugándolos con los requerimientos científicos de calidad y profundidad de los datos requeridos para la ejecución de acciones de manejo.

Pasos metodológicos: La lógica del trabajo en el área se orientó en dos vías complementarias entre sí:

1. La elaboración y ejecución de una metodología para la realización de estudios de evaluación global sobre el estado de todos los ecosistemas de humedales existentes en el área.

2. El desarrollo de una metodología propia de elaboración de planes de manejo, adaptada a las condiciones de la zona y la ejecución de un plan de manejo piloto en un área de humedales a identificarse.

Los trabajos iniciales incluyeron la realización de una serie de sondeos para tener un primer acercamiento a la realidad del territorio, ya que la información secundaria existente no servía, como consecuencia del cambio radical que experimentó toda la zona tras el paso del huracán Juana en 1988. Como información secundaria, se consideró en su momento toda la información que existía sobre el área: mapas de cobertura vegetal, informes de uso de recursos, población asentada, etc.

Se identificó una parte del Sistema de Humedales de la Bahía de Bluefields que podía ser considerada una unidad en sí por sus características físicas (se ubica en el sector más alejado del mar y presenta procesos naturales que permiten diferenciarla del resto del Sistema). Se trata de los Humedales asociados a los ríos Mahogany y Caño Negro (Castrillo, 1997; Castrillo, 1998).

A partir de los sondeos iniciales a la zona, se recopiló información que sirvió para identificar los vacíos de información y orientar los trabajos técnicos para llenarlos.

Principios considerados en el proceso de elaboración e implementación del Plan de Manejo

$\checkmark$ Participación: Se consideró, desde un inicio, que la participación de los usuarios y dueños de los recursos en là planificación y ejecución de cualquier esfuerzo para el uso racional de los recursos naturales era un factor primordial para mantener en el tiempo las acciones de manejo, ya fueran de preservación, de uso o de desarrollo. Los procesos a implementarse debían ser conocidos por los usuarios de los recursos del humedal, y debían participar en todos ellos. La información técnica a recopilar debía ser presentada y discutida con la población usuaria de los recursos, y con las instituciones con presencia y autoridad en la zona, de manera que los productos de los diferentes trabajos a implementarse fueran el resultado de la suma de los procesos técnicos, más el conocimiento empírico de quienes históricamente han usado el recurso. 
$\checkmark$ La información técnica como soporte de equivalencia entre los actores: Se considera que la participación es óptima cuando cada uno de los actores cuenta con el mismo nivel de información "comprendida" sobre cualquier tópico de discusión. En este sentido, siempre orientados a garantizar niveles equivalentes de participación entre los actores, se consideró la producción de información técnica sobre los recursos y los procesos en los ecosistemas y sus amenazas, su posterior traducción a lenguaje popular y la entrega a cada uno de los actores.

$\checkmark$ La consideración de toda la población usuaria en la toma de decisiones sobre el área: La realidad de la zona indica que sobre los recursos de los humedales de Mahogany / Caño Negro, existen dos grupos que ejercen presión y se deben considerar como usuarios (agentes externos y los pobladores asentados en las áreas).

$\checkmark$ Desarrollo y ejecución de un Plan sobre la marcha: Al considerar la participación de las poblaciones como base para la implementación de un Plan de Manejo, y considerando que no se puede esperar demasiado tiempo para emprender acciones en las áreas por el riesgo de perderlas, se propuso el desarrollo paulatino del proceso para la elaboración de un Plan de Manejo que consideró: En primer lugar, la participación de las comunidades en la planificación de sus territorios y en la administración de las medidas a implementarse. En segundo lugar, el respeto a los tiempos normales de acción de los usuarios, por sobre los tiempos de los técnicos que acompañan el proceso, en la ejecución de las acciones. En tercer lugar, la ejecución de acciones de manejo identificadas como urgentes y acordadas a partir de discusiones entre los usuarios y las instituciones, utilizando como insumos los elementos que aportan los estudios técnicos básicos. Desde este concepto, el Plan de Manejo se elabora y se ejecuta sobre la marcha. De este modo, se garantiza la participación sostenida de los diferentes usuarios y se evita el "cansancio" que los procesos excesivamente técnicos provocan en las poblaciones. Esta concepción de elaboración de Planes de Manejo retarda la elaboración y presentación de un documento, pero acelera la ejecución de las acciones del Plan, asegurando altos niveles de participación local lo que, a la postre, puede garantizar de mejor manera la permanencia de los procesos de planificación y manejo en el tiempo por los compromisos adquiridos en el proceso por los diferentes actores.(Castrillo, 2000).

\section{Fases de ejecución}

$\checkmark$ Primera fase: Recopilación de información básica sobre la situación de elementos clave de los ecosistemas y de los niveles de presión humana sobre estos.

Segunda fase: Comparación de información local sobre los procesos y usos del área con la información técnica. Con información de los avances de los trabajos técnicos, se desarrolla una serie de discusiones sobre los valores, problemas y posibles acciones a tomarse en los territorios. 
$\checkmark$ Tercera fase: Elaboración de una propuesta de Plan transitorio, en conjunto con la comunidad y las instituciones. Toma de decisiones perentorias de manejo del área. En este momento, debe esperarse la toma de decisiones por parte de los usuarios que normalmente no chocarán con las acciones posteriores que, con más información técnica, se decidan en el área. En el caso de chocar, no harán más que afinar el Plan General de Manejo.

$\checkmark$ Cuarta fase: Identificación de áreas críticas. Este proceso requiere de trabajo técnico de gabinete. Con la información provista por los usuarios y los trabajos técnicos, se tiene la posibilidad de identificar, con el apoyo de diversos métodos de ordenamiento de la información, las diferentes áreas críticas teóricas (problemas de conservación y potencialidades de uso) y especiales (identificación de diferentes territorios especiales de manejo). Con esta información, se puede desarrollar la siguiente fase.

$\checkmark$ Quinta fase: Elaboración de una propuesta de pre-Plan. Inicio de discusiones entre usuarios y autoridades con competencia en el área. Elaboración técnica de una propuesta de pre zonificación con su reglamentación, sistema de seguimiento y los elementos básicos de monitoreo de dinámica de ecosistemas. En este momento, es indispensable la presencia de las diversas autoridades e instituciones clave para proseguir el desarrollo del proceso, se empieza a tener más clara la ejecución de los pasos a seguir, principalmente cómo administrar el área. Está etapa posibilita la toma de compromisos por las autoridades con competencia en el área y la llegada a consenso con las diversas representaciones de organizaciones locales, con interés en el manejo del área.

$\checkmark$ Sexta fase: Elaboración de una propuesta final de documento. Seguimiento de los procesos legales del país para la presentación y aprobación legal de un Plan de Manejo de un Área Protegida.

\section{Resultados}

En la actualidad se tienen como principales resultados, los siguientes:

- Acciones de manejo aplicándose en el área, sobre la marcha del proceso de elaboración del Plan de Manejo:

1. Prohibición en la zona de caza y pesca con fines comerciales.

2. La prohibición del uso de venenos en sus cuerpos de agua.

3. La conformación de guardaparques voluntarios.

4. La conformación de policía voluntaria en cada una de sus comunidades

5. El rotulado de sus áreas.

- Resolución municipal apoyando las acciones de manejo decididas por los usuarios del área. 
- Delimitación y zonificación del área.

- Grupo de guardaparques comunitarios (sin devengar salario) conformado y operando con el reconocimiento legal de las autoridades.

- Establecidas alianzas inter-comunales, ejecutando acciones para el desarrollo de sus territorios.

- Alcaldías con competencia en el área coordinada para la presentación de Plan de Manejo.

- Una propuesta de normativa municipal declarando la zona como "Parque Ecológico Municipal", mientras el Plan es aprobado por los niveles nacionales y es reconocida una categoría más idónea.

- Documento de Plan de Manejo presentado por un grupo de actores claves y en proceso de aprobación legal en el ámbito nacional.

- Ficha Ramsar de la bahía elaborada, presentada por el gobierno de Nicaragua ante la convención en Ginebra y aprobada por la convención.

- Plan de Acción Municipal de la Alcaldía de Bluefields en proceso de aprobación.

En general, se considera que por el nivel y calidad de la participación garantizada por el método de ejecución de este Plan de Manejo, puede ser utilizado como modelo para ser reproducido en el resto del Sistema de Humedales de la Bahía de Bluefields.

\section{Referencias bibliográficas}

-CASTRILLO, M. (1997). Metodología de Evaluaciones Globales de Ecosistemas en el Área de Cerro Silva. PROCODEFOR. Bluefields, Nicaragua.

-CASTRILLO, M. (1998). Propuesta de Elaboración de un Plan de Manejo para los Humedales de Mahogany/Caño Negro. PROCODEFOR. Bluefields Nicaragua.

-CASTRILLO, M. (2000). Plan de Manejo de los Humedales de Mahogany: primera propuesta de zonificación. Revista Trimestral Humedales de la RAAS, No. 3:1-18. Lugar y fecha

-CASTRILLO, M, RAMOS, Z., y KARREMANS., J., (2000). Ficha Técnica Ramsar, del Sistema de Humedales de la Bahia de Bluefields. PROCODEFOR, Bluefields, Nicaragua. 